\title{
A Comparison of Reconstruction Algorithms Regarding Exposure Dose Reductions during Digital Breast Tomosynthesis
}

\author{
Tsutomu Gomi \\ School of Allied Health Sciences, Kitasato University, Sagamihara, Japan \\ Email: gomi@kitasato-u.ac.jp
}

Received 8 April 2014; revised 25 May 2014; accepted 8 June 2014

Copyright (C) 2014 by author and Scientific Research Publishing Inc. This work is licensed under the Creative Commons Attribution International License (CC BY). http://creativecommons.org/licenses/by/4.0/

c) (i) Open Access

\section{Abstract}

This study compared reconstruction algorithms [filtered back projection (FBP) and simultaneous iterative reconstruction technique (SIRT)] with respect to radiation doses and image quality and suggested the possibility of decreasing the exposure dose in digital breast tomosynthesis (DBT). These two existing algorithms were implemented using a DBT system and experimentally evaluated using contrast-detail (CD) phantom measurements, such as contrast-to-noise ratio (CNR), root mean square error (RMSE), intensity profile, and artifact spread function (ASF), and the results obtained with FBP and SIRT were compared. The potential dose reduction, contrast improvement, quantum noise reduction, and artifact reduction in DBT were evaluated using different exposures and the two reconstruction techniques. The effectiveness of each technique for enhancing the visibility of a CD phantom was quantified with respect to CNR and RMSE, and artifact reduction was quantified with respect to the intensity profile and ASF. SIRT produced reconstructed images with CNR values indicative of high-contrast detection. Image error was smaller in the in-focus plane SIRT images, and artifacts were decreased in these images according to the determined intensity profiles and ASF. These results suggest that when using SIRT, the exposure dose could possibly be decreased to half.

\section{Keywords}

Digital Breast Tomosynthesis, 3-Dimensional Reconstruction, Radiation Dose

\section{Introduction}

High-resolution imaging digital mammography can successfully detect masses and microcalcifications, which

How to cite this paper: Gomi, T. (2014) A Comparison of Reconstruction Algorithms Regarding Exposure Dose Reductions during Digital Breast Tomosynthesis. J. Biomedical Science and Engineering, 7, 516-525. 
are markers of interest during breast cancer screening. Despite the success of mammography for early breast cancer detection, this method is associated with superposition issues because of its 2-dimensional (2D) acquisition geometry. Digital breast tomosynthesis (DBT) aims to overcome these issues by generating images with limited-angle tomographic projections.

Tomosynthesis refers to a limited-angle image reconstruction method in which a dataset of projections acquired at regular intervals during a single acquisition pass is used to reconstruct planar sections post priori. Tomosynthesis slices have high resolution in planes parallel to the detector plane. Furthermore, tomosynthesis provides the additional benefits of digital imaging [1] [2], as well as the tomographic benefits of computed tomography (CT) at a reduced radiation dose and costs, in an approach that can easily be implemented in conjunction with radiography. However, these images are invariably affected by blurring, which is caused by objects that lie outside the plane of interest but are superimposed on the focused fulcrum plane image by the limited acquisition angle. This blurring can result in poor object detectability in the in-focus plane.

DBT is among the most promising techniques for the improvement of early breast cancer detection [2]-[5] because it can provide 3-dimensional (3D) structural information by reconstructing an entire image volume from a sequence of projection-view mammograms acquired at a small number of projection angles over a limited angular range, and the total radiation dose is comparable with that used during regular mammography screening. DBT has been shown [6] to decrease the camouflaging effect of the overlapping fibroglandular breast tissue, thus improving the conspicuity of subtle lesions. Several digital mammography-based DBT systems have been developed [7], and preliminary pilot clinical studies are ongoing to evaluate the utility of this technique [2] [8].

Various DBT reconstruction methods have been explored previously [3] [9]-[11]. However, image quality assessments have been based on the use of phantoms with features that did not address radiation doses. In fact, to date, no studies have quantitatively compared DBT algorithms with respect to image quality and radiation dose. One recent CT technique, iterative reconstruction (IR), was found to effectively decrease quantum noise and radiation exposure [12]. IR might yield improvements in image quality and exposure dose reduction relative to those associated with the conventional filtered back projection (FBP) technique. In this study, we chose to focus on the simultaneous iterative reconstruction technique (SIRT) [13]. This DBT study evaluated and compared the reconstructed image qualities and possible exposure dose reduction associated with the FBP and SIRT algorithms. The algorithms were implemented using a DBT system and experimentally evaluated through contrast-detail (CD) phantom measurements.

\section{Materials and Methods}

\subsection{DBT}

The DBT system (Selenia Dimensions, Hologic Inc., Bedford, MA, USA) comprised an X-ray tube with a 0.3-mm focal spot and a $240 \times 290-\mathrm{mm}$ digital flat-panel amorphous selenium detector. Each detector element was $70 \times 70 \mu \mathrm{m}$ in size. Tomography was performed using a linear tomographic movement, a total acquisition time of $3.7 \mathrm{~s}$, and an acquisition angle of $15^{\circ}$. Projection images were sampled during a single tomographic pass (15 projections) and used to reconstruct tomograms of a desired height. Reconstructed images $(0.1 \mathrm{~mm} / \mathrm{pixel})$ were obtained at $1-\mathrm{mm}$ reconstruction intervals. The distance between the isocenter and the detector was 700 $\mathrm{mm}$ (0.7-mm aluminum-equivalent filtration).

\subsection{Reconstruction Algorithm}

In FBP algorithms, which are widely used in tomography, many projections are acquired for cross-sectional image reconstruction. In 2D tomographic imaging, object projecting corresponds to sampling perpendicular to the $\mathrm{X}$-ray beam in the Fourier space [9]. The relationship between the radon transform and cone-beam projections has been thoroughly studied, and cone-beam reconstruction solutions have been provided [9]. 2D image filtering via multiplication of the Fourier transform by a Ramp or Shepp-Logan (SL) filter kernel restores the proper impulse shape to the reconstructed image. Filtration in a 2D Fourier space comprises an acceptable image restoration method, but a much more rapid equivalent filtering response might be obtained by filtering the 1-dimensional projection data prior to back projection. The FBP algorithm generally provides highly precise 3D reconstruction images [9]. In this study, a conventional SL filter kernel was used to reconstruct the FBP images.

IR algorithms perform reconstruction recursively [10] [11], unlike the 1-step operation used in back projection and FBP algorithms. Instead, reconstruction is accomplished by iteratively updating unknown linear attenu- 
ation coefficients through minimization of the error between the measured and calculated projection data.

The original method in this family of algebraic reconstruction techniques (ARTs) [13] has already been determined. ART features a fast convergence speed because only a single projection value is used to update linear attenuation coefficients at a given time point, but it converges to a least-squares solution that can result in considerable noise when severely ill-posed inverse problems such as limited-angle reconstruction are addressed. Variations have been proposed regarding ART implementation in order to facilitate improvements. ART can be modified in accordance with other methods such as SIRT [13], depending on the amount of projection data and the method used to update the current estimation. SIRT is applied iteratively such that reconstructed volume projections, which are computed from an image formation model, resemble the experimental projections. In this study, 10 SIRT iterations were used for image quality.

FBP and SIRT image reconstruction processing from real DBT system projection data was performed using MATLAB (Mathworks, Natick, MA, USA) [14].

\subsection{Phantom Specifications}

A CD phantom and polymethyl methacrylate (PMMA) slabs were used for the evaluation of low-contrast resolution. $\mathrm{CD}$ phantoms of different diameters (artificial region, $\mathrm{CaCO}_{3}$ ) and thicknesses were arranged within the PMMA slabs (Figure 1). For X-ray imaging, we arranged the PMMA slabs $(150 \times 150 \mathrm{~mm}, 100-\mathrm{mm}$ thickness) on the top and bottom of the CD phantom.

\subsection{Exposure Dose Measurement}

Each radiation dose setup was acquired with the following settings: a reference exposure dose [automatic exposure control $=$ the exposure condition of a 40-mm phantom (CD phantom + PMMA phantom) and determined tube voltage and tube current values] of $28 \mathrm{kVp}, 50 \mathrm{mAs}$; a half-exposure dose of $28 \mathrm{kVp}, 24 \mathrm{mAs}$; and a quarter-exposure dose of $28 \mathrm{kVp}, 12 \mathrm{mAs}$. All target and filter combinations comprised tungsten (W) and rhodium (Rh).

We calculated the average glandular dose (AGD) according to the method suggested by Dance et al. [15]. For 2D breast examinations, the dose $D$ was calculated as follows:

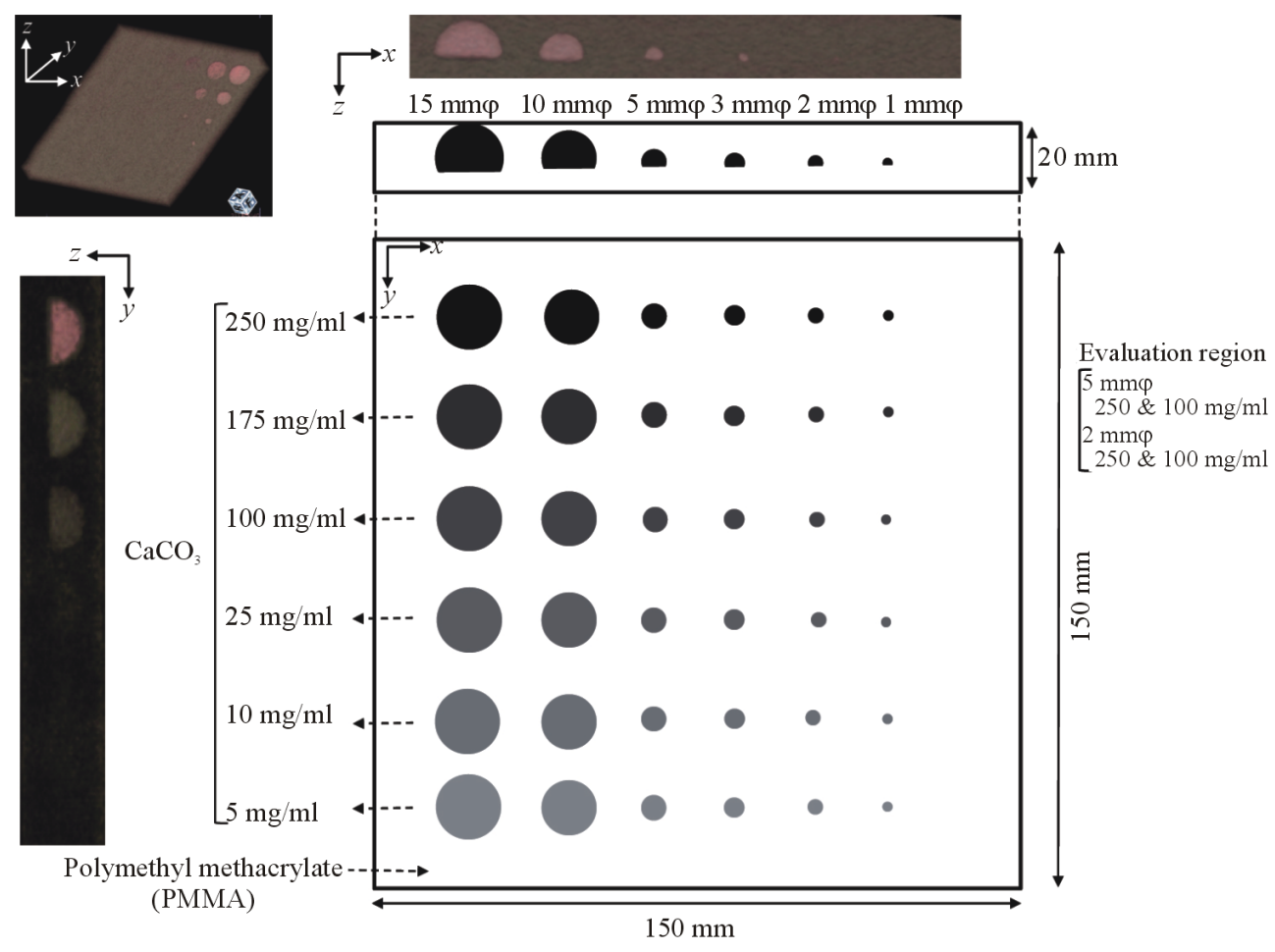

Figure 1. Illustration of the contrast-detail (CD) phantom used in this study. 


$$
D=k_{g c s},
$$

where $k$ was the incident air kerma at the upper surface of the breast, measured without backscatter from the breast, and $g, c$, and $s$ were conversion factors. For 3D breast examinations, the dose $D_{T}$ was calculated as follows:

$$
D=k_{\text {Tgcs }} T .
$$

Here, the incident air kerma, $K_{T}$, was again measured in the "straight through" position, but this time it takes into account the tube current during the examination; $T$ was a conversion factor. A Piranha radiation exposure measurement device was used (RTI Electronics AB, Sweden). The purpose of the exposure dose measurement was to convert the established exposure condition (mAs) into AGD (mGy). Results of AGD were as follows: for the reference exposure dose (thickness $30 \mathrm{~mm} ; 1.78 \mathrm{mGy}$, thickness $40 \mathrm{~mm} ; 1.51 \mathrm{mGy}$, thickness $50 \mathrm{~mm} ; 1.29$ mGy, thickness $60 \mathrm{~mm} ; 1.13 \mathrm{mGy}$ ); for the half-exposure dose (thickness $30 \mathrm{~mm} ; 0.93 \mathrm{mGy}$, thickness $40 \mathrm{~mm}$; $0.78 \mathrm{mGy}$, thickness $50 \mathrm{~mm}$; $0.67 \mathrm{mGy}$, thickness $60 \mathrm{~mm}$; $0.59 \mathrm{mGy}$ ); and for the quarter-exposure dose (thickness $30 \mathrm{~mm} ; 0.48 \mathrm{mGy}$, thickness $40 \mathrm{~mm} ; 0.40 \mathrm{mGy}$, thickness $50 \mathrm{~mm} ; 0.34 \mathrm{mGy}$, thickness $60 \mathrm{~mm} ; 0.30$ mGy).

\subsection{Image Contrast}

All experiments (for image contrast, error image, and artifact evaluation) were performed according to the arrangement shown in Figure 2. To quantitatively evaluate the quality of the reconstructed image, we calculated the image contrast derived from the contrast-to-noise ratio (CNR) [16] of selected features to determine lowand high-contrast detectability (regions, $\varphi 2$ and $\varphi 5 \mathrm{~mm} ; \mathrm{CaCO}_{3}, 250$ and $100 \mathrm{mg} / \mathrm{ml}$ ) in the in-focus plane. CNR was defined as follows:

$$
C N R=\frac{N_{1}-N_{0}}{\sigma_{0}},
$$

where $N_{1}$ was the mean object pixel value, $N_{0}$ was the mean background area pixel value, and $\sigma_{0}$ was the standard deviation of the background pixel values. The parameter $\sigma_{0}$ not only included the photon statistics and electronic noise from the results but also the structural noise that might obscure the object. The sizes of all regions of interest (ROIs) were set up to a signal internally. ROI areas of measurement for the CNR metric are presented in Figure of CNR results ( $\varphi 5 \mathrm{~mm}$ : ROI area 104 pixels, $\varphi 2 \mathrm{~mm}$ : ROI area 9 pixels).

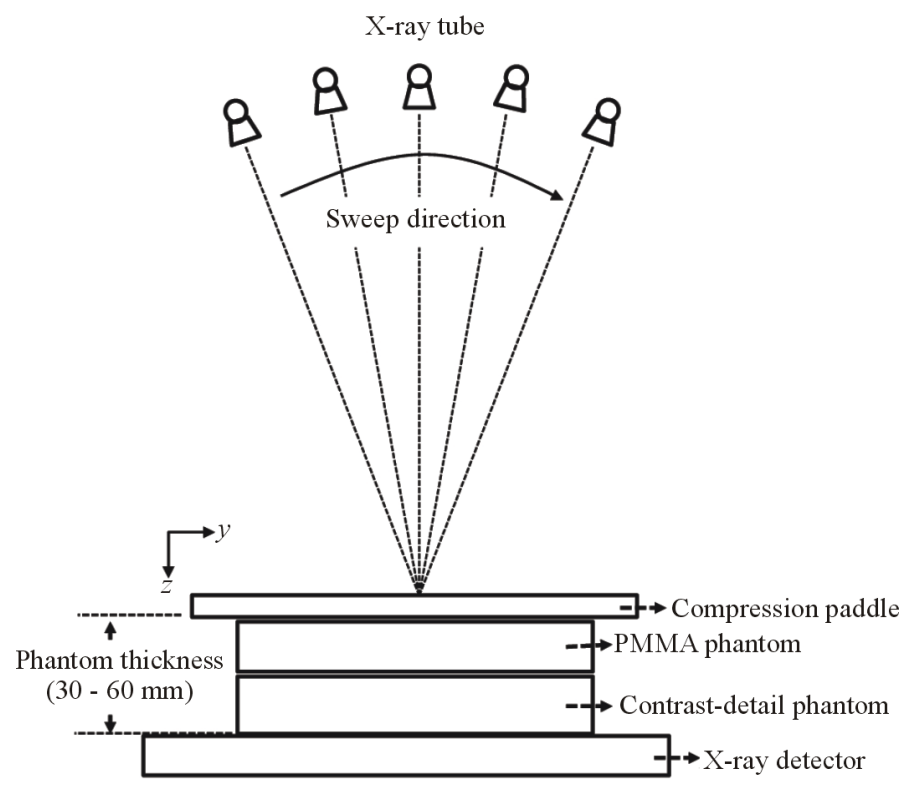

Figure 2. For digital breast tomosynthesis acquisition, the phantom was arranged parallel to the $x-y$ detector plane. 


\subsection{Error Image Differences}

Another important metric to consider is the root mean square error (RMSE), which can be computed by obtaining the root of the summed squares of the standard deviation and bias. The image plane errors were defined in terms of RMSE as follows:

$$
R M S E=\sqrt{\sum_{i=1}^{n}\left(X-x_{i}\right)^{2} / n},
$$

where $X$ was the observed image, $x_{i}$ was the reference image, and $n$ was the number of compounds in the analyzed set. In this study, the reference images were reference dose reconstructed images, the observed images used to compare RMSE were half- and quarter-dose reconstructed images in the in-focus plane.

\subsection{Artifacts}

To evaluate the effects of artifacts on the reconstructed image features in the adjacent out-of-plane (z direction) area, we calculated the artifact spread function (ASF). Different in-focus plane reconstruction methods (region: $\varphi 5 \mathrm{~mm} ; 250 \mathrm{mg} / \mathrm{ml} \mathrm{CaCO}_{3}$ ) were used to compare intensity profiles. Wu et al. proposed an ASF metric that would quantify the artifacts observed in planes outside the focus image plane [17]. These artifacts, which resemble real features, are generated from real features observed within the focus image plane. The ability of DBT to differentiate superimposed features along the $\mathrm{z}$ direction might be reflected in the ASF measurement. The artifacts exhibited in the image plane can be defined by ASF as follows:

$$
A S F=\frac{N_{\text {artifact }}(z)-N_{B G}(z)}{N_{\text {artifact }}\left(z_{0}\right)-N_{B G}\left(z_{0}\right)},
$$

where $z_{0}$ and $z$ represent the in-focus plane and out-of-plane locations of the real features, respectively; $N_{\text {artifact }}\left(z_{0}\right)$ and $N_{B G}\left(z_{0}\right)$ represent the average pixel intensity ROIs of the artifact and image background in the in-focus plane, respectively; and $N_{\text {artifact }}(z)$ and $N_{B G}(z)$ represent the average pixel intensity ROIs of the artifact and image background in the out-of-plane, respectively. A ROI size of $2 \times 6$ pixels was chosen for the evaluations of all features.

\section{Results}

We compared the intensity profiles, CNRs, RMSEs, and ASFs of reconstructed images obtained using different exposure conditions with FBP and SIRT. The results revealed that SIRT produced reconstructed images with high-contrast detectable features with no artifacts in the vertical direction (X-ray sweep direction). A review of the results revealed that both DBT artifact reduction and high-contrast $(250 \mathrm{mg} / \mathrm{ml})$ resolution were highest with SIRT, but low-contrast (100 mg/ml) resolution can be seen better with FBP at the lowest dose level (Figure 3).

However, the contrast resolution was higher in the FBP images than in the SIRT images. The high-contrast and large-diameter $(5 \mathrm{~mm})$ characteristics of SIRT yielded an increase in the in-focus plane CNR values, whereas the low-contrast and small-diameter $(2 \mathrm{~mm})$ characteristics yielded equivalent in-focus plane CNR values. Using FBP, the reference dose detectability rates in the CNR experiment were approximately equivalent to those of the high-contrast and large-diameter characteristic half-dose images generated using SIRT. Comparisons of the CNR values at different exposure doses revealed that the improved results required exposure above that of a half dose because the CNR value for the half-dose SIRT image was close to that of the reference dose image. This result suggests that the exposure dose might possibly be decreased to half (Figure 4, Figure 5).

The RMSE values (Table 1) revealed a reduction in vertical direction (X-ray sweep direction) artifacts with SIRT, and this technique more effectively decreased quantum noise in the half-dose images. In addition, these results indicated that the improved artifact reduction with SIRT was unrelated to the exposure dose. For 40-, 50-mm, and 60-mm phantom thickness, the FBP images had higher RMSE values (60-mm: reference between quarter only), and for 30- and 60-mm thickness, the SIRT images had higher RMSE values (60-mm: reference between half only).

The intensity profiles of the FBP and SIRT images are presented in Figure 6 (upper). Artifacts were decreased with SIRT, and the consequent improvements in image quality (related to the signal undershoot) were demonstrated. The charts in Figure 6 (lower) present the ASF results for the CD phantom. The charts demon- 
FBP

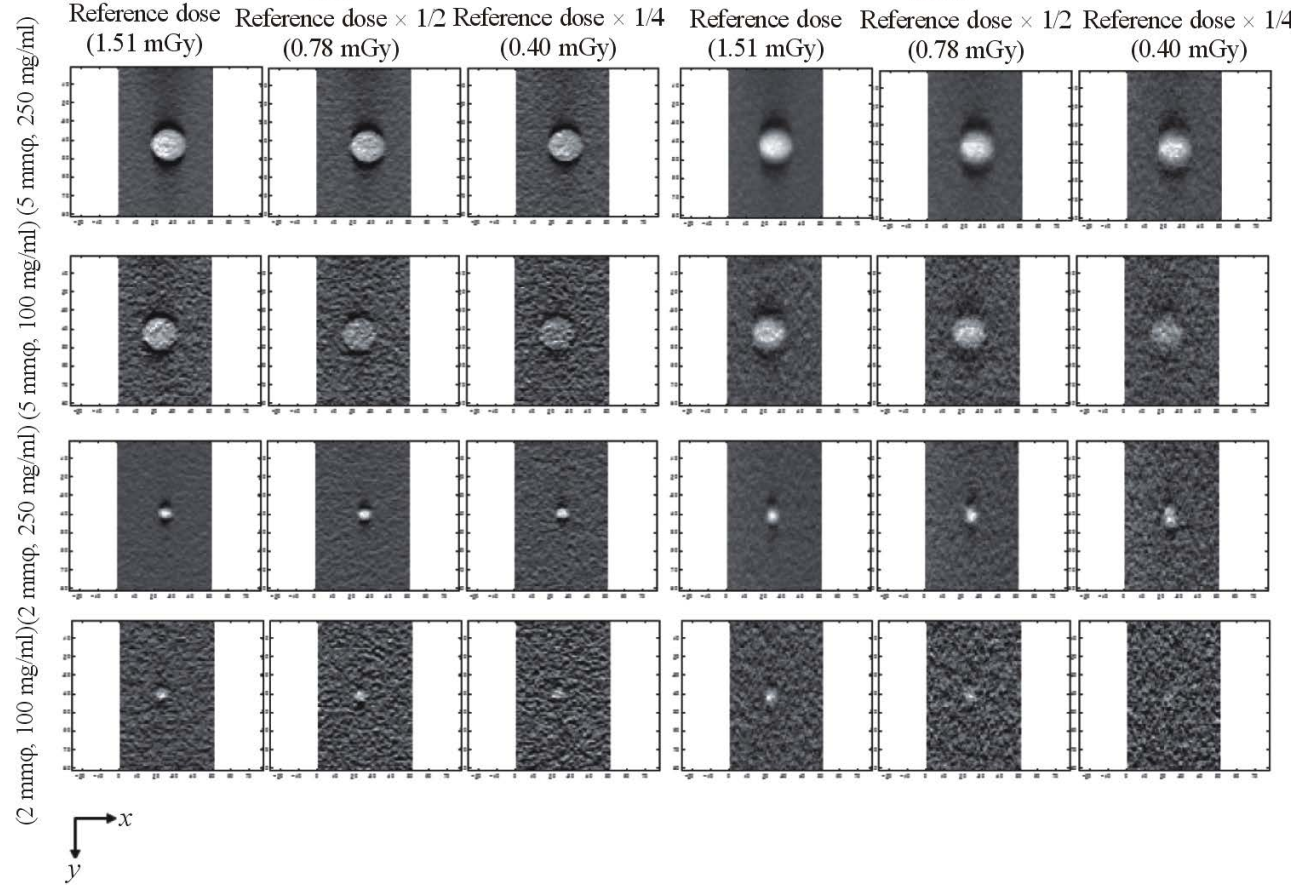

Figure 3. A comparison of digital breast tomosynthesis images and images obtained using the filtered back projection (FBP) and simultaneous iterative reconstruction technique (SIRT) reconstruction algorithms in the in-focus plane (phantom thickness: $40 \mathrm{~mm}$ ).

FBP $5 \mathrm{~mm} \varphi, 250 \mathrm{mg} / \mathrm{ml}$

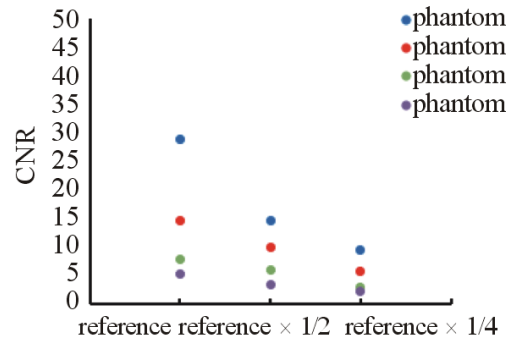

FBP $5 \mathrm{~mm} \varphi, 100 \mathrm{mg} / \mathrm{ml}$

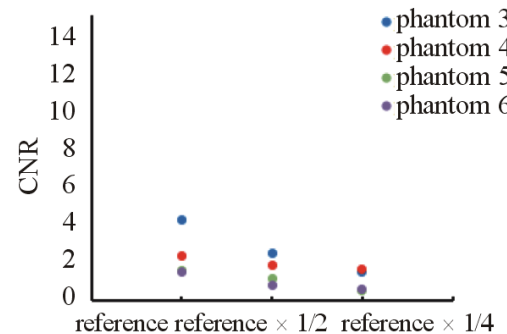

SIRT $5 \mathrm{~mm} \varphi, 250 \mathrm{mg} / \mathrm{ml}$

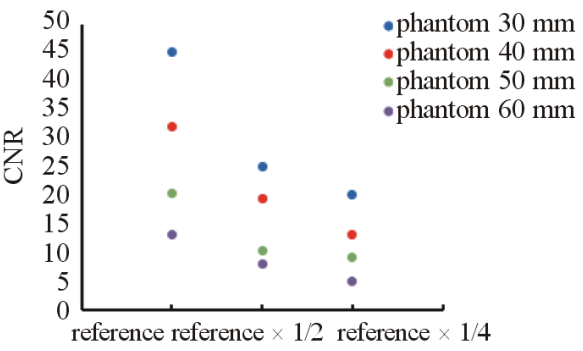

SIRT $5 \mathrm{~mm} \varphi, 100 \mathrm{mg} / \mathrm{ml}$

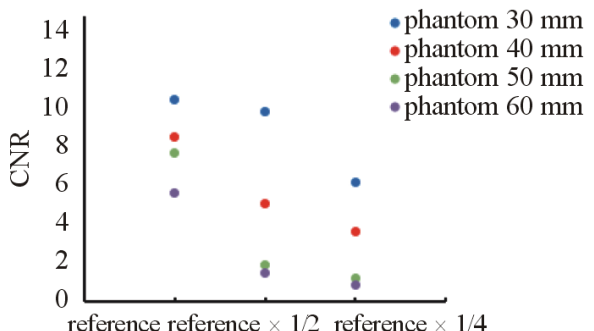

Figure 4. Comparison of the contrast-to-noise ratio (CNR) values obtained using digital breast tomosynthesis in the in-focus plane $(\varphi 5 \mathrm{~mm}, 100$ and $250 \mathrm{mg} / \mathrm{ml})$ for different phantom thicknesses and exposures. The simultaneous iterative reconstruction technique (SIRT) was used; the contrast detectability obtained with this technique was higher than that obtained with the filtered back projection (FBP) technique. 
FBP $2 \mathrm{~mm} \varphi, 250 \mathrm{mg} / \mathrm{ml}$

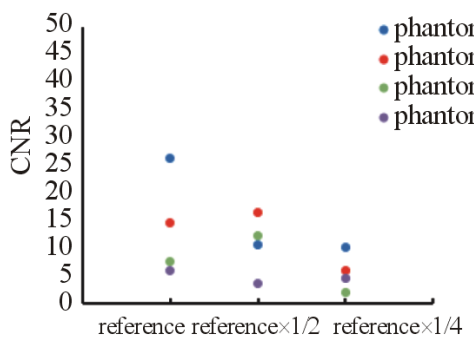

FBP $2 \mathrm{~mm} \varphi, 100 \mathrm{mg} / \mathrm{ml}$

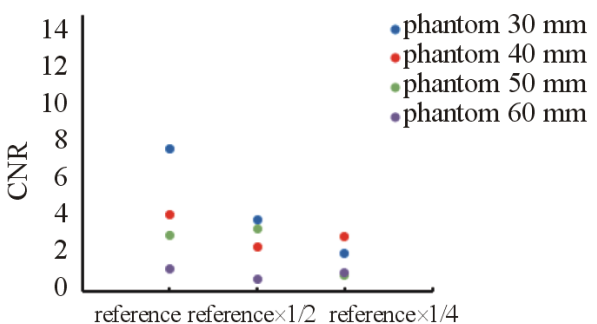

SIRT $2 \mathrm{~mm} \varphi, 250 \mathrm{mg} / \mathrm{ml}$

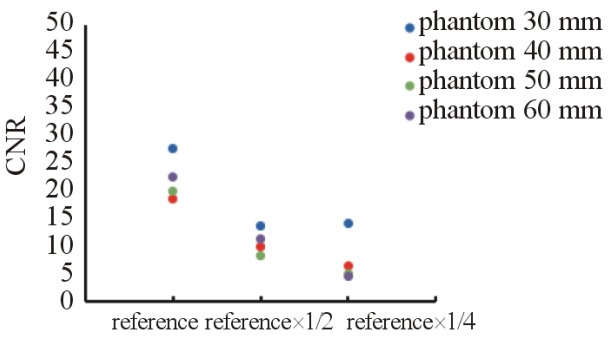

SIRT $2 \mathrm{~mm} \varphi, 100 \mathrm{mg} / \mathrm{ml}$

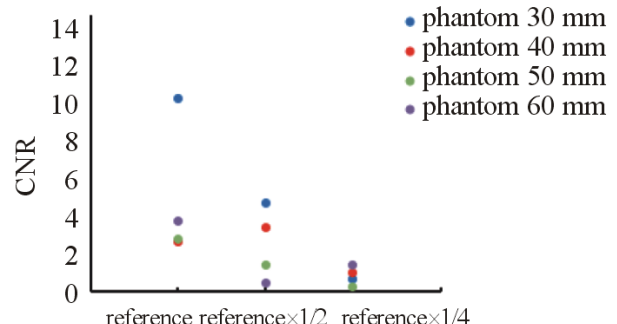

Figure 5. Comparison of the contrast-to-noise ratio (CNR) values obtained using digital breast tomosynthesis in the in-focus plane ( $\varphi 2 \mathrm{~mm}, 100$ and $250 \mathrm{mg} / \mathrm{ml}$ ) for different phantom thicknesses and different exposures. The simultaneous iterative reconstruction technique (SIRT) was used; the contrast detectability obtained with this technique was higher than that obtained with the filtered back projection (FBP) technique.
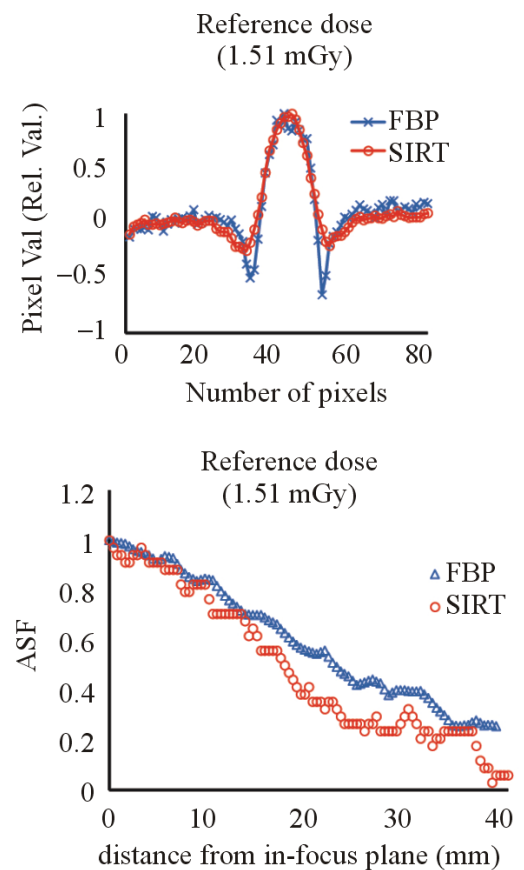

Reference dose $\times 1 / 2$ $(0.78 \mathrm{mGy})$
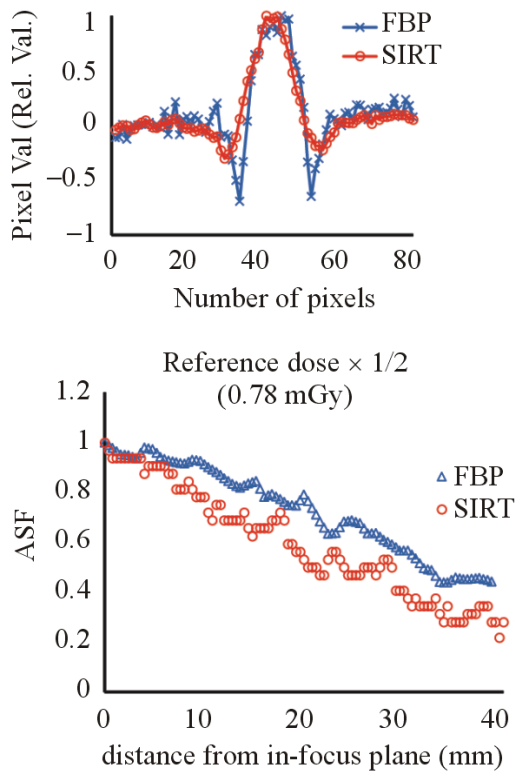

Figure 6. Upper: Comparison of the in-focus plane intensity profiles using different reconstruction algorithms and different exposures $(\varphi 5 \mathrm{~mm}, 250 \mathrm{mg} / \mathrm{ml}$, phantom thickness: $40 \mathrm{~mm}$ ). Lower: Comparison of the artifact spread function (ASF) versus the distance from the in-focus plane using different reconstruction algorithms and different exposures ( $\varphi 5 \mathrm{~mm}, 250 \mathrm{mg} / \mathrm{ml}$, phantom thickness: $40 \mathrm{~mm}$ ). 
Table 1. Comparison of the root mean square error (RMSE) values using two digital breast tomosynrhesis (DBT) algorithm (FBP vs. SIRT) for different phantom thickness.

\begin{tabular}{ccccc}
\hline & \multicolumn{3}{c}{ RMSE } \\
\cline { 2 - 5 } & \multicolumn{2}{c}{ FBP } & \multicolumn{2}{c}{ SIRT } \\
\hline Phantom thickness & reference - reference $\times 1 / 2$ & reference - reference $\times 1 / 4$ & reference - reference $\times 1 / 2$ & reference - reference $\times 1 / 4$ \\
\hline $30 \mathrm{~mm}$ & 0.019 & 0.026 & 0.039 & 0.064 \\
$40 \mathrm{~mm}$ & 0.073 & 0.174 & 0.037 & 0.041 \\
$50 \mathrm{~mm}$ & 0.111 & 0.159 & 0.037 & 0.081 \\
$60 \mathrm{~mm}$ & 0.067 & 0.138 & 0.095 & 0.086 \\
\hline
\end{tabular}

strate that the artifacts were removed when DBT incorporated SIRT. Better image quality with respect to artifact reduction was demonstrated with SIRT than with FBP.

\section{Discussion}

Our experimental results clearly demonstrate the possibility that the exposure dose can be decreased to half in low-dose DBT images using the evaluated SIRT. For example, the efficiency of this technique could be quantitatively assessed on the basis of intensity profiles, CNR, RMSE, and ASF, which are presented in Figures 3-6 and Table 1. In the CNR experiment, the detectable contrast in the reference dose (FBP images) and half-dose images obtained using SIRT were approximately equivalent; therefore, this technique could potentially decrease the DBT exposure dose.

Results of the CNR experiment are not in complete agreement with the visual impression of Figure 3. One reason may be that CNR is a zero frequency measure, and for visual impression, higher frequencies play a role. In DBT FBP, low-frequency contributions are largely suppressed, but visually perceived acuity might be good because of some level of edge enhancement.

At a given projection angle, an attenuation feature can create artifacts in any voxel along lines between the $\mathrm{X}$-ray source and the projection feature pixels. Artifact reduction becomes more difficult with an increase in the attenuation feature size or a decrease in the distance between the voxel and the attenuation feature. SIRT does not assume even noise distribution across the entire image. Instead, an algebraic matrix is used to selectively identify and then subtract noise from the image according to a mathematical model. The result is a less noisy image, which is an unexpected effect of image artifact reduction.

In general, observed artifacts in an image are caused by the loss of the largest normal contributions from artifact-free voxels. These voxels have normal original contributions, and their values are slightly decreased after the largest normal contribution is omitted. A voxel with a single abnormal contribution rejects this contribution while retaining all other contributions, including the largest normal contribution. Therefore, these voxels tend to exhibit higher values than their neighboring artifact-free voxels, leading to the appearance of objects (especially high-contrast), in which artifact-free voxels are more noticeable in the background. This phenomenon is a drawback of the FBP technique, but artifacts due to this effect are very evident when such images are compared with artifact-free images.

In DBT reconstruction, the noise mask is calculated as the sum of the restored set of all DBT plane-blurred replicas that have been weighted accordingly. Therefore, this sum contains the image, which is added to the plane of interest as quantum noise. However, this sum also contains a directionally blurred version of the tomogram of interest. The edges of the reconstructed objects in the plane of interest are enhanced in the direction of the line that intersects the tomogram with the trajectory plane.

There were some potential limitations in our phantom study. First, the materials constituting the phantom were pure PMMA, and the research was not performed using real mammary gland tissues. However, we believe that the consistency of PMMA is an accurate representation of real mammary gland tissue. Second, the aim of this phantom study was to evaluate the low-contrast resolution. Our results have limitations, but we suggest that they can be used as reference data in the consideration of reducing radiation exposure.

In conclusion, our experimental results clearly demonstrate that SIRT can be used to improve image contrast by effectively removing quantum noise and suppressing streak artifacts in DBT images obtained using both reference and half-exposure doses. However, low-contrast resolution can be seen better perceived with FBP at the 
lowest dose level. Using SIRT, the radiation levels could be decreased to approximately half of that used for the FBP technique.

\section{Acknowledgements}

We thank Yukio Koibuchi, M.D., Ph.D. and Katsuya Fujita, R.T. of the National Hospital Organization Takasaki General Medical Center for invaluable assistance with the study.

\section{References}

[1] Sone, S., Kasuga, T., Sakai, F., Kawai, T., Oguchi, K., Hirano, H., Li, F., Kubo, K., Honda, T., Hniuda, M., Takemura, K. and Hosoba, M. (1995) Image Processing in the Digital Tomosynthesis for Pulmonary Imaging. European Radiology, 5, 96-101.

[2] Skaane, P., Bandos, A.I., Gullien, R., Eben, E.B., Ekseth, U., Haakenaasen, U., Izadi, M., Jebsen, I.N., Jahr, G., Krager, M., Niklason, L.T., Hofvind, S. and Gur, D. (2013) Comparison of Digital Mammography Alone and Digital Mammography plus Tomosynthesis in a Population-Based Screening Program. Radiology, 267, 47-56. http://dx.doi.org/10.1148/radiol.12121373

[3] Niklason, L.T., Christian, B.T., Niklason, L.E., Kopans, D.B., Castleberry, D.E., Opsahl-Ong, B.H., Landberg, C.E., Slanetz, P.J., Giardino, A.A., Moore, R., Albagli, D., DeJule, M.C., Fitzgerald, P.F., Fobare, D.F., Giambattista, B.W., Kwasnick, R.F., Liu, J., Lubowski, S.J., Possin, G.E., Richotte, J.F., Wei, C.Y. and Wirth, R.F. (1997) Digital Tomosynthesis in Breast Imaging. Radiology, 205, 399-406.

[4] Wu, T., Stewart, A., Stanton, M., McCauley, T., Phillips, W., Kopans, D.B., Moore, R.H., Eberhard, J.W., Opsahl-Ong, B., Niklason, L. and Williams, M.B. (2003) Tomographic Mammography Using a Limited Number of Low-Dose Cone-Beam Projection Images. Medical Physics, 30, 365-380. http://dx.doi.org/10.1118/1.1543934

[5] Rafferty, E.A., Park, J.M., Philpotts, L.E., Poplack, S.P., Sumkin, J.H., Halpern, E.F. and Niklason, L.T. (2013) Assessing Radiologist Performance Using Combined Digital Mammography and Breast Tomosynthesis Compared with Digital Mammography Alone: Results of a Multicenter, Multireader Trial. Radiology, 266, 104-113. http://dx.doi.org/10.1148/radiol.12120674

[6] Helvie, M.A., Roubidoux, M.A., Zhang, Y., Carson, P.L. and Chan, H.P. (2006) Tomosynthesis Mammography vs Conventional Mammography: Lesion Detection and Reader Reference. Initial Experience. RSNA Program Book, 335.

[7] Sechopoulos, I., Bliznakova, K. and Fei, B. (2013) Power Spectrum Analysis of the X-Ray Scatter Signal in Mammography and Breast Tomosynthesis Projections. Medical Physics, 40, 101905-1-101905-7.

[8] Gur, D., Zuley, M.L., Anello, M.I., Rathfon, G.Y., Chough, D.M., Ganott, M.A., Hakim, C.M., Wallace, L., Lu, A. and Bandos, A.I. (2012) Dose reduction in digital breast tomosynthesis (DBT) screening Using Synthetically Reconstruction Projection Images: An Observer Performance Study. Academic Radiology, 19, 166-171. http://dx.doi.org/10.1016/j.acra.2011.10.003

[9] Dobbins 3rd, J.T. and Godfrey, D.J. (2003) Digital x-Ray Tomosynthesis: Current State of the Art and Clinical Potential. Physics in Medicine and Biology, 48, R65-R106. http://dx.doi.org/10.1088/0031-9155/48/19/R01

[10] Bleuet, P., Guillemaud, R., Magnin, I. and Desbat, L. (2001) An Adapted Fan Volume Sampling Scheme for 3D Algebraic Reconstruction in Linear Tomosynthesis. IEEE Transactions on Nuclear Science, 3, 1720-1724.

[11] Wu, T., Zhang, J., Moore, R., Rafferty, E. and Kopans, D. (2004) Digital Tomosynthesis Mammography Using a Parallel Maximum-Likelihood Reconstruction Method. Proceedings of SPIE, 5368, 1-11. http://dx.doi.org/10.1117/12.534446

[12] Marin, D., Nelson, R.C., Schindera, S.T., Richard, S., Youngblood, R.S. and Yoshizumi, T.T. (2010) Low-TubeVoltage, High-Tube-Current Multidetector Abdominal CT: Improved Image Quality and Decreased Radiation Dose with Adaptive Statistical Iterative Reconstruction Algorithm-Initial Clinical Experience. Radiology, 254, 145-153. http://dx.doi.org/10.1148/radiol.09090094

[13] Gordon, R., Bender, R. and Hermen, G.T. (1970) Algebraic Reconstruction Techniques (ART) for Three-Dimensional Electron Microscopy and X-Ray Photography. Journal of Theoretical Biology, 29, 471-481. http://dx.doi.org/10.1016/0022-5193(70)90109-8

[14] Mathworks Inc. (2014) http://www.mathworks.com/products/matlab/

[15] Dance, D.R., Young, K.C. and van Engen, R.E. (2011) Estimation of Mean Glandular Dose for Breast Tomosynthesis: Factors for Use with the UK, European and IAEA Breast Dosimetry Protocols. Physics in Medicine and Biology, 56, 453-471. http://dx.doi.org/10.1088/0031-9155/56/2/011

[16] Welvaert, M. and Rosseel, Y. (2013) On the Definition of Signal-To-Noise Ratio and Contrast-To-Noise Ratio for 
FMRI Data. PLoS One, 8, Article ID: e77089. http://dx.doi.org/10.1371/journal.pone.0077089

[17] Wu, T., Stewart, A., Stanton, M., McCauley, T., Phillips, W. and Kopans, D.B. (2003) Tomographic Mammography Using a Limited Number of Low-Dose Cone-Beam Projection Images. Medical Physics, 30, 365-380. http://dx.doi.org/10.1118/1.1543934

\section{Abbreviations}

DBT Digital breast tomosynthesis

FBP Filtered back projection

SIRT Simultaneous iterative reconstruction technique

CNR Contrast-to-noise ratio

RMSE Root mean square error

ASF Artifact spread function 
Scientific Research Publishing (SCIRP) is one of the largest Open Access journal publishers. It is currently publishing more than 200 open access, online, peer-reviewed journals covering a wide range of academic disciplines. SCIRP serves the worldwide academic communities and contributes to the progress and application of science with its publication.

Other selected journals from SCIRP are listed as below. Submit your manuscript to us via either submit@scirp.org or Online Submission Portal.
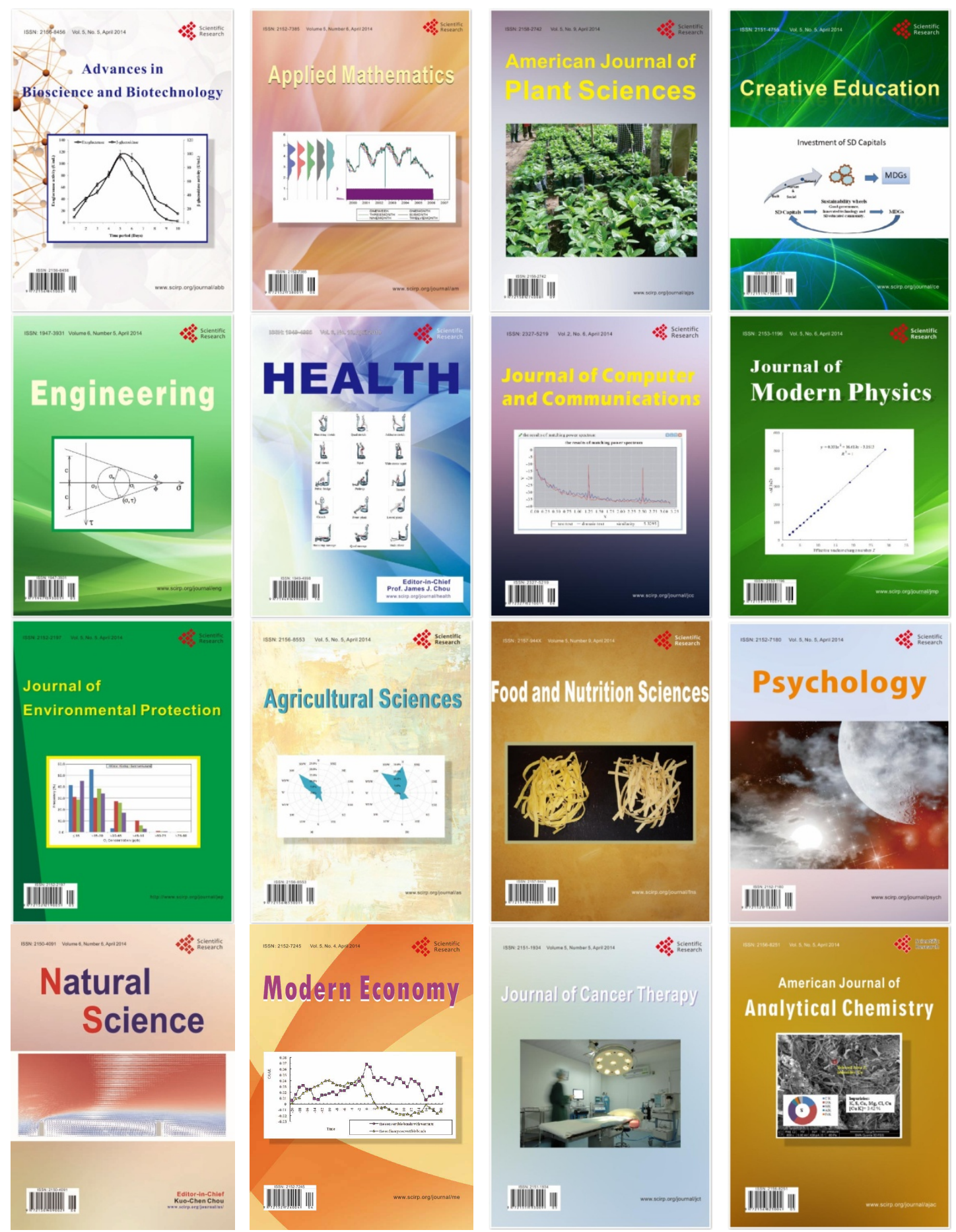